\title{
Research of Possibilities of Synthesis of Optical and Radar Data in Flood Space Monitoring
}

\author{
Oleg P. Arkhipkin, Gulshat N. Sagatdinova \\ National Centre for Space Research and Technology, Almaty, Kazakhstan \\ oarkhipkin@rambler.ru
}

\begin{abstract}
The paper describes the possibility of using the fusion (synthesis) of optical and radar satellite images of medium resolution for the formation and analysis of water surfaces. The method of formation of synthesized images of optical and radar data at the object level including separate formation of water surfaces by optical and radar data and their subsequent joint analysis is presented. The technique of formation of the synthesized images of optical and radar data at the point level, including their geometrical binding to each other, selection of the method of data fusion, synthesis and their analysis is also described. The methods of fusion of optical and radar data are illustrated by the example of passing a flood in 2017 on the Ishim river in the districts of the city of Petropavlovsk in North Kazakhstan region and the village of Gastello in Akmola Oblast.
\end{abstract}

Keywords: space monitoring, flood, water surfaces, flood zones, radar data, optical data, fusion 


\title{
ИССЛЕДОВАНИЕ ВОЗМОЖНОСТЕЙ СИНТЕЗА ОПТИЧЕСКИХ И РАДАРНЫХ ДАННЫХ ПРИ КОСМИЧЕСКОМ МОНИТОРИНГЕ ПАВОДКОВ
}

\author{
О.П. Архипкин, Г.Н. Сагатдинова
}

Национальный центр космических исследований и технологий, Алма-Ата, Казахстан oarkhipkin@rambler.ru

В работе описываются возможности использования слияния (синтеза) оптических и радарных космоснимков среднего разрешения для формирования и анализа водных поверхностей. Приводится методика формирования синтезированных изображений оптических и радарных данных на уровне объектов, включающая раздельное формирование водных поверхностей по оптическим и радарным данным и последующий их совместный анализ. Описана также методика формирования синтезированных изображений оптических и радарных данных на точечном уровне, включающая их геометрическую привязку друг к другу, выбор метода слияния данных, синтез и их анализ. Методы слияния оптических и радарных данных проиллюстрированы на примере прохождения паводка 2017 года на реке Ишим в районах города Петропавловска Северо-Казахстанской области и поселка Гастелло Акмолинской области.

Ключевые слова: космический мониторинг, паводки, водные поверхности, зоны затопления, радарные данные, оптические данные, синтез

\section{Введение}

Практически каждый год происходят ЧС различного масштаба на больших и малых реках Казахстана в период прохождения паводковых вод. Методы космического мониторинга являются наиболее эффективным способом обнаружения и контроля развития значительного числа природных и техногенных ЧС на водных объектах. Космический мониторинг паводков и наводнений включает в себя задачи оперативной динамики схода снежного покрова и прохождения паводковых вод. В отдельную задачу выделяется космический мониторинг динамики заполнения важнейших водохранилищ, а также ряда других водных объектов.

При прохождении паводковых вод главная задача мониторинга состоит в выделении зон затопления. Зоны затопления определяются как разность текущей водной поверхности и водной поверхности в период минимального уровня воды, который считаем в качестве постоянной водной поверхности. Он формируются по осенним данным Landsat-8. Определение зон затопления осуществляется в трех уровнях. Первоначально регулярно формировались зоны затопления только по данным низкого разрешения. После появления в свободном доступе оптических данных Landsat-8 и Sentinel-2 A, а также радарных данных Sentinel-1 A и Sentinel-1 В стало возможным регулярно формировать зоны затопления и по данным среднего разрешения. 
В Казахстане НЦКИТ с 2002 года разрабатывает технологии космического мониторинга паводков и наводнений, которые одновременно внедрялись в практическую деятельность органов ЧС различного уровня $[1,2]$. В последние годы активно развиваются методы и технологии космического мониторинга паводков и наводнений на основе комплексного анализа оптических и радарных ДДЗ различного разрешения с активным использованием указанных выше новых данных среднего и высокого разрешения [3], а также методов радарной поляриметрии [4] и синтеза радарных и оптических данных, рассматриваемых в данной работе.

\section{Цели, основные понятия и методы слияния разнородных данных Д33}

Основная цель формирования синтезированных изображений разнородных ДДЗ состоит в повышении информативности космического мониторинга и в повышении качества получаемых в результате слияния выходных данных, учитывая преимущества и недостатки каждого из ДДЗ. Данные ДЗ3 различаются между собой по спектральным характеристикам, по радиометрическому разрешению, по пространственному разрешению, по времени съемки, сенсорами, с которых их получают, природой (типом) данных (оптические, радарные, СВЧ).

Одним из первых и наиболее ярким и успешным примером синтеза разнородных ДДЗ является слияние разных по спектральным характеристикам данных, полученных одновременно с помощью одного сенсора, то есть каналов многоспектрального снимка. Методика такого слияния обычно базируется на формировании $\mathrm{rgb}$-композита из разных каналов одного космоснимка. Другим примером такого успешного и широко применяемого слияния является синтез PAN и MX снимков, полученных одним и тем же сенсором. Методика такого слияния часто базируется на процедуре Pansharpening.

Практический интерес к слиянию разнородных данных ДЗ возник давно, практически сразу, как только приступили к решению конкретных прикладных задач [5]. В настоящее время разработаны разнообразные методы слияния разнородных ДД3, в том числе радарных и оптических [6-9]. Слияние радарных и оптических данных используется при классификации подстилающей поверхности в картографии, сельском и лесном хозяйствах, ирригации, при геологических и гидрологических исследованиях, городской инфраструктуры и т.д.

Имеются примеры использования этих методов и при космическом мониторинге ЧС (последствия землетрясений [9], снежный и ледовый покров [9], выгоревшие площади [10], зоны прибрежного затопления [11], оползни [9]). Совместное использование радарных и оптических данных применялось при космическом мониторинге динамики развития паводков с помощью их комплексного анализа [12], на уровне объектов [13] и с помощью Байесовской сети [14].

Алгоритмы слияния данных оптических и радиолокационных сенсоров могут быть условно разбиты на три группы: 1) уровень элементов изображения (точечный уровень), 2) уровень объектов, 3) уровень решений (интерпретации). На точечном уровне осуществляется слияние данных на уровне пикселей, например с помощью rgb композита. На уровне объектов вначале проводится раздельное формирование водных поверхностей по оптическим и радарным данным, а затем их совместный анализ. Эти два уровня будут рассмотрены ниже.

\section{Формирование синтезированных изображений оптических и радарных данных на уровне объектов}

Алгоритм слияния данных оптических и радарных сенсоров на уровне объектов включает в себя раздельное формирование водных поверхностей по оптическим и радарным данным и последующий их совместный анализ. При слияния оптических и радарных данных на этом уровне выбираем один какой-либо вариант формирования водных поверхностей для каждого из них, получаем по ним маски водных поверхностей, проводим их совместный анализ и получаем итоговую синтезированную водную поверхность.

Функционирование методики слияния оптических и радарных данных на уровне объектов рассмотрим на тестовом примере для фрагмента района поселка Гастелло (рисунок 1). На этом 
рисунке представлен один и тот же фрагмент реки Ишим на радарном (VV поляризация) и оптическом (rgb композит) исходных снимках. Видно, что пространственное расположение зон затопления примерно одинаково. Видна также схожая внутренняя структура в зоне затопления, повторяющая русло реки, только на оптическом снимке она менее выражена. При формировании водной поверхности эта структура исчезает на оптическом снимке и остается на радарном (рисунок 2).
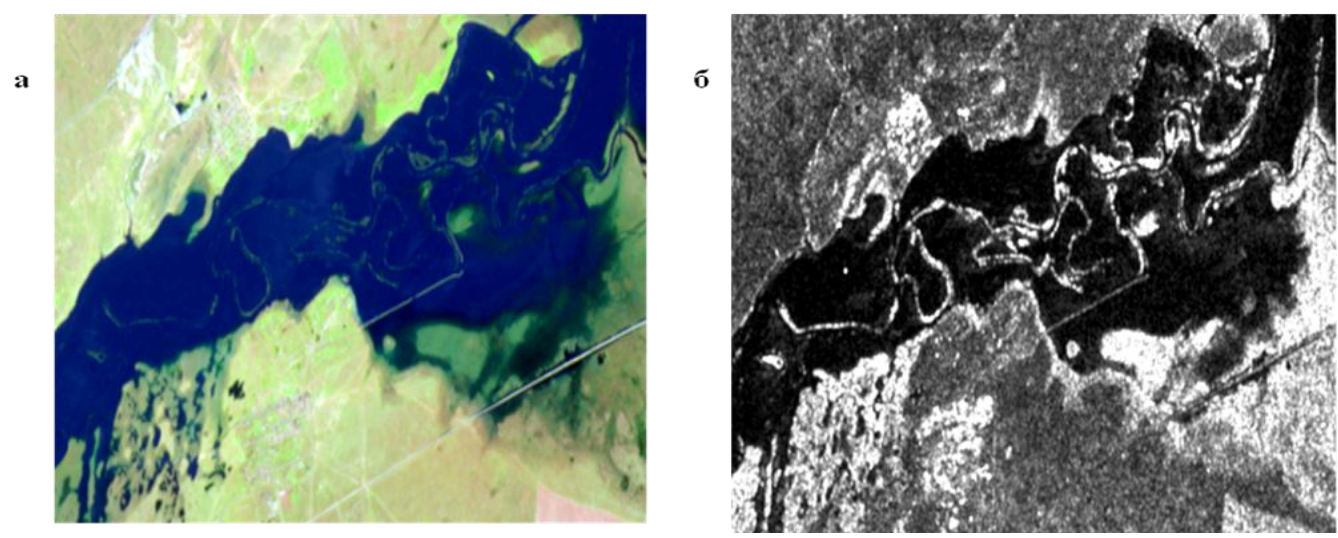

Puc. 1. Фрагмент оптического Sentinel-2 (а) и радарного Sentinel-1В (б) снимков района поселка Гастелло за 1 мая 2017 года
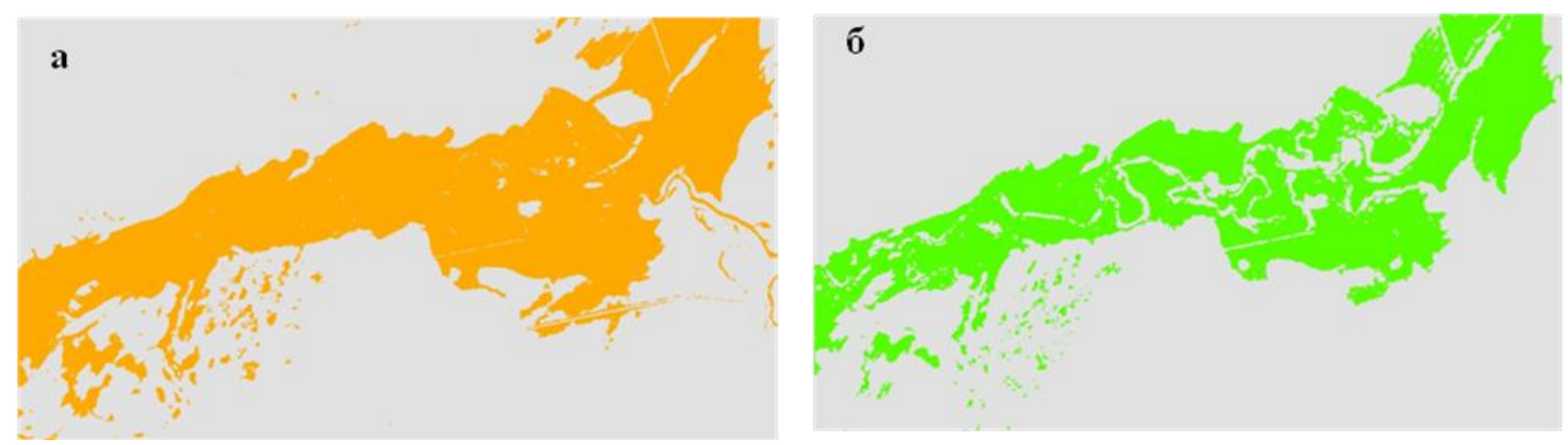

Puc.2. Выделение водной поверхности по оптическим (а) и радарным (б) данным на реке Ишим в районе поселка Гастелло Акмолинской области за 1 мая 2017 года

Совместный анализ водных поверхностей, полученных по оптическим и радарным данным (рисунок 2) позволяет провести структурирование затопленной территории. В результате слияния оптических и радарных данных на уровне объектов выделяем водную поверхность и затопленную растительность. При этом вектор воды берем с оптического снимка, так как при одинаковом разрешении оптические данные дают более качественную водную поверхность. Затопленную растительность формируем на основе радарных данных с учетом оптических данных о незатопленных островах внутри затопленной территории. В результате получаем синтезированную по радарным и оптическим данным маску воды (рисунок 3 ).

Предположение о том, что выделенный на рисунке 3 класс представляет собой затопленную растительность, первоначально основывалось на его расположении вдоль русла реки в обычном состоянии, вдоль которого часто растет прибрежная растительность. Это предположение позже нашло подтверждение на снимках высокого разрешения (порядка 1 м), полученных в Google Earth Engine (рисунок 4). На них четко видно, что растительность располагается узкой полосой вдоль русла, утолщаясь в некоторых местах, но все равно занимая небольшие площади.

Качество выделения затопленной растительности по радарным данным за 1 мая иллюстрирует рисунок 4, в котором вектор затопленной растительности, полученный по синтезированным данным за 1 мая 2017 года фрагмента полигона Гастелло, наложен красным цветом на космоснимок высокого разрешения. Учитывая разницу в пространственном разрешении со снимком высокого разрешения (20 и 1 м соответственно), можно утверждать, что 
затопленная растительность по результатам синтеза оптических и радарных данных на уровне объекта выделилась достаточно хорошо.

Слияние оптических и радарных данных можно использовать и для выделения других классов объектов, включая имеющий существенное значение при мониторинге паводков объект мокрые почвы. При этом можно использовать и больше двух снимков.

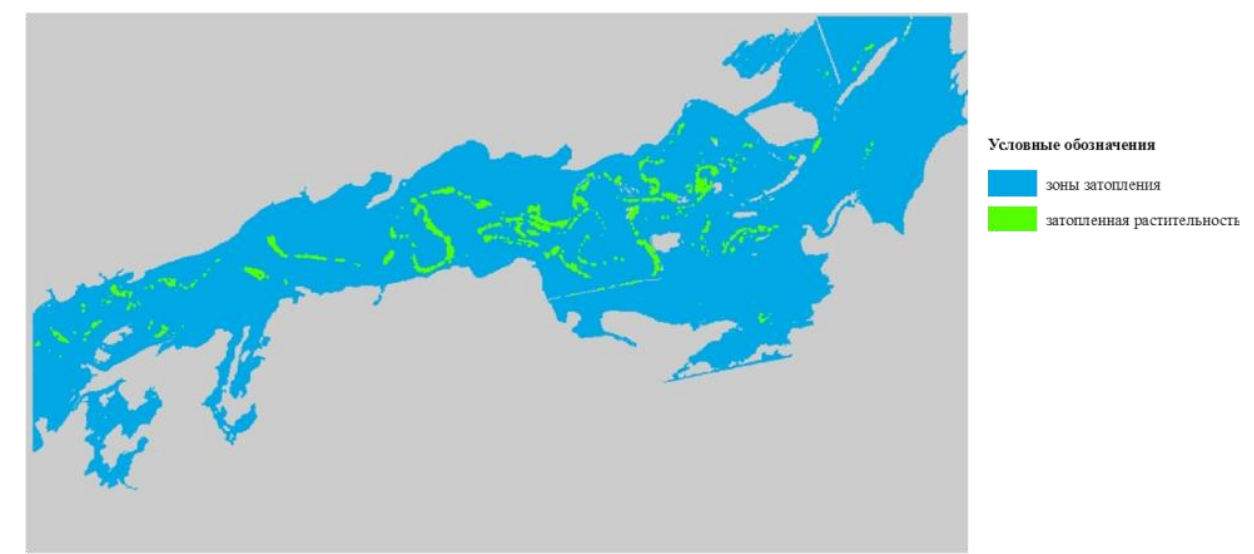

Puc. 3. Комбинированная маска воды, полученная при слиянии радарных данных Sentinel-1 и оптических Sentinel-2 за 1 мая 2017 года

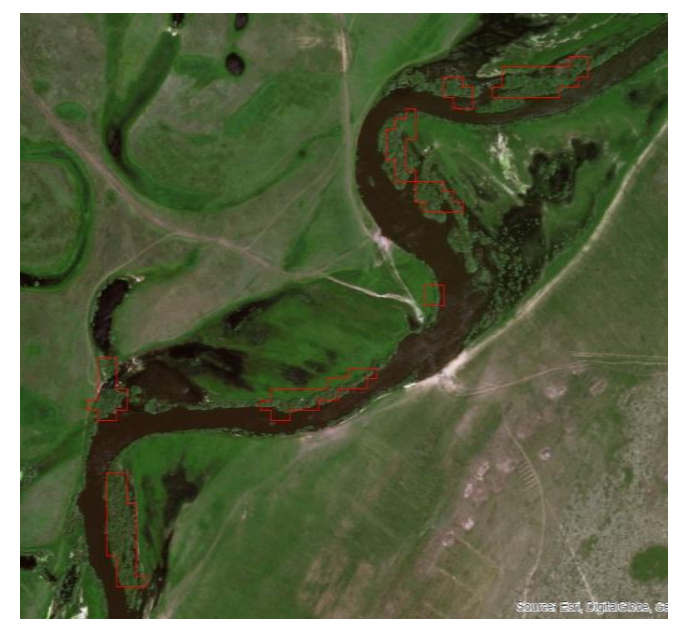

Puc.4. Вектор затопленной растительности, полученный в результате синтеза радарных и оптических данных за 1 мая 2017 года фрагмента полигона Гастелло, наложенный красным цветом на космоснимок высокого разрешения

\section{Формирование синтезированных изображений оптических и радарных данных на точечном уровне}

Алгоритм слияния оптических и радарных данных на точечном уровне состоит из следующих процедур: геометрическая привязка оптических и радарных данных друг к другу, выбор комбинации оптических и радарных данных при синтезе, синтез оптических и радарных данных.

Процедура геометрической привязки оптических и радарных данных друг к другу отрабатывалась в свободно распространяемом программном обеспечении SNAP (The Sentinel Application Platform). Этот программный комплекс может работать с любыми ДДЗ, но прежде всего предназначен для работы с данными спутников Sentinel. Отметим, что используемые оптические данные Sentinel-2 и Landsat имеют очень хорошую привязку. Проблемы были с привязкой радарных данных Sentinel-1. Эта проблема решалась при введении вместо 
оперативных орбитальных данных уточненных орбитальных данных. Тогда и привязка радарных данных соответствовала привязке оптических данных.

Использование синтезированных данных очень полезно для устранения ошибок радарных и оптических данных. Ошибки радарных и оптических данных обусловлены их особенностями. Ошибки радарных данных вызваны зернистостью радарных снимков, условиями съёмки (угол визирования, прозрачность атмосферы, облачность), наличием плоских участков. При этом имеются ошибки и первого, и второго рода: часть водных пикселей воспринимается как не водные и наоборот не водные воспринимаются как водные. Ошибки оптических снимков обусловлены прежде всего наличием облачности и дымки, которые не позволяют провести качественную съемку подстилающей поверхности, экранируемой ими.

Исследование формирования синтезированных изображений оптических и радарных данных на точечном уровне проведено на примере районов поселка Гастелло и Петропавловска. При этом рассмотрены случаи уменьшения ошибок как в случае радарных данных, так и оптических. Исследования начали с синтеза радарных и оптических данных, полученных в один день. Таких дат было несколько, но наиболее качественные оптические снимки обоих полигонов получены 1 мая 2017 года. Водные поверхности, выделенные по радарным данным за эту дату, содержали много ошибочно выделенных водных поверхностей, особенно для полигона Петропавловск (рисунок 5).

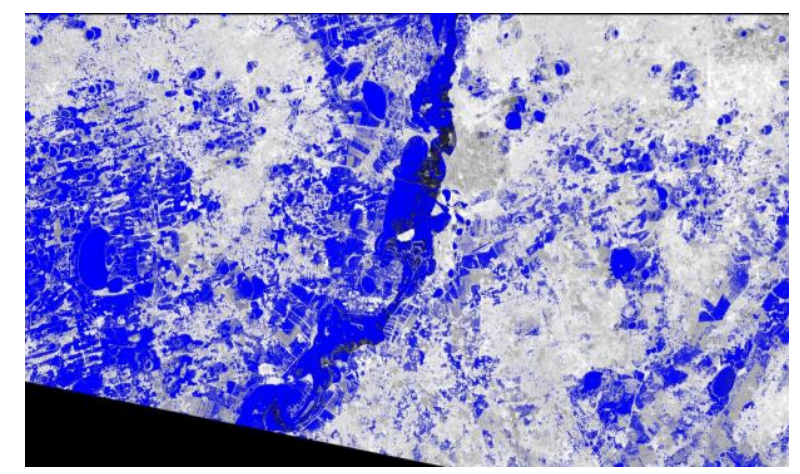

Puc. 5. Вектор воды, полученный по снимку Sentinel-1 за 1 мая 2017 года для полигона Петропавловск, наложенный на маску NDVI

Для уменьшения таких ошибок были сформированы синтезированные изображения оптических и радарных данных. Синтез можно осуществлять с помощью формирования различных rgb композиций из каналов оптических и радарных данных Так водная поверхность на трех из них (VV NDVI MI), (VV b11 b8), (b11 VH b4) близка к оптическим данным. Здесь bi каналы оптических данных Sentinel-2, вегетационный индекс NDVI $=(\mathrm{b} 8-\mathrm{b} 4) /(\mathrm{b} 8+\mathrm{b} 4)$, индекс влажности $\mathrm{MI}=(\mathrm{b} 8 \mathrm{~A}-\mathrm{b} 11) /(\mathrm{b} 8 \mathrm{~A}+\mathrm{b} 11)$. Но можно синтез осуществить с помощью совместного анализа только радарных и оптических индексов. Так, на рисунке 6 представлена маска воды, полученная путем совместного анализа радарного параметра шероховатость (VV-VH) и оптического SWB (Soils and Water bodies = b2/b11), которая практически идентична оптической.

Для практических целей важна возможность использования при синтезе оптические и радарные снимки, полученные в разные, но близкие даты. Продемонстрируем это для случая, когда радарный снимок позволяет откорректировать оптический. На рисунке 7 приведен результат формирования водной поверхности по снимку Landsat-8, значительная часть которой покрыта облачностью и дымкой, в результате чего значительная ее часть не идентифицируется.

Для этой же территории имеется радарный снимок Sentinel-1 за 24 апреля. Используем его для корректировки водной поверхности полученной по оптическим данным (рисунок 7). Слияние данных проводим через совместный анализ радарного параметра шероховатость и оптического SWB. По результатам анализа сформирована водная поверхность, представленная на рисунке 8. Она содержит в себе и водную поверхность, находящуюся под облачным покровом. Для избегания потери данных в случае спада паводка такой анализ проводится только на территории покрытой облачностью, используя маску облачности. 


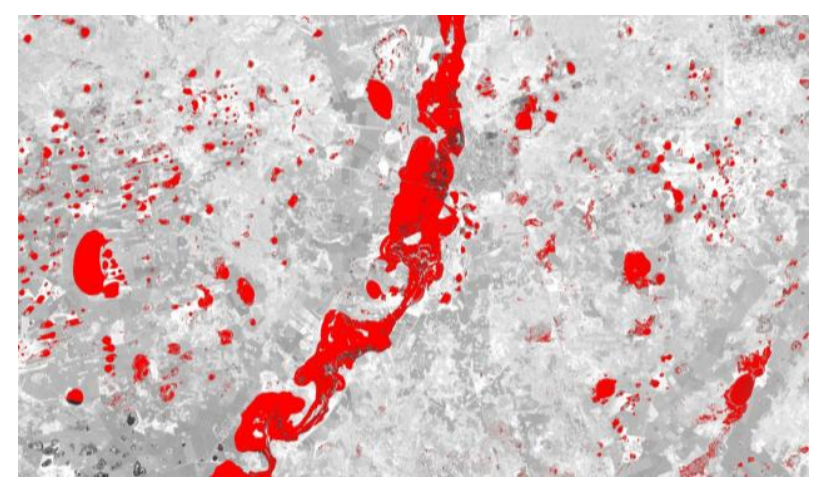

Рuc.6. Вектор воды, полученный путем совместного анализа радарного параметра шероховатость и оптического SWB, полученных по снимку Sentinel-1 и 2 за 1 мая 2017 года для полигона Петропавловск, наложенный на маску NDVI

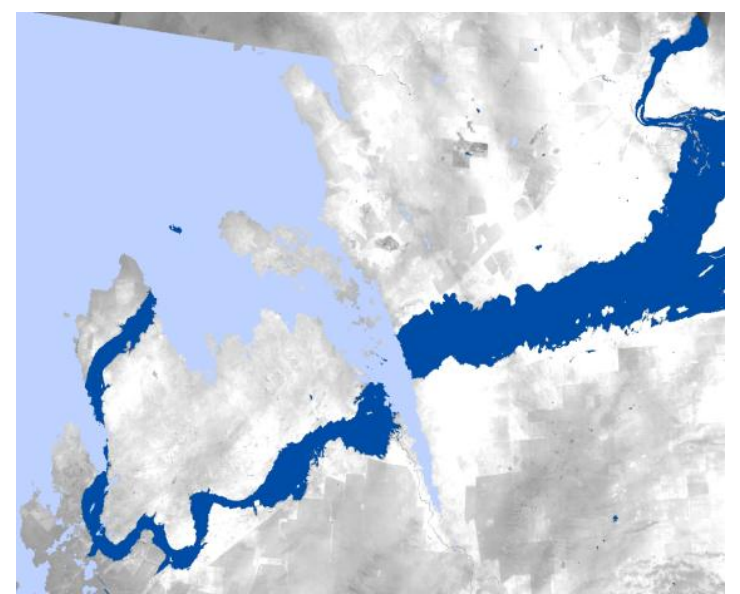

Puc. 7. Вектор воды, полученный по снимку Landsat-8 за 26 апреля 2017 года для полигона Гастелло, наложенный на маску NDVI

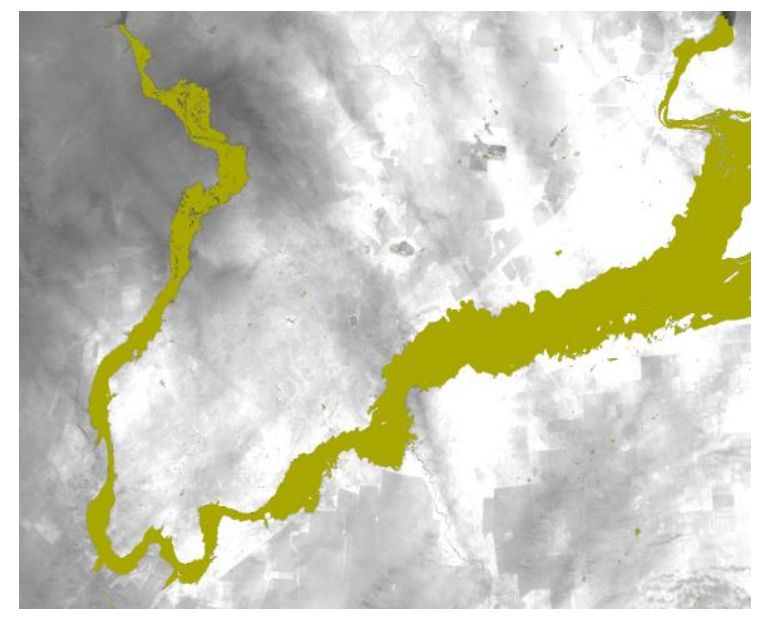

Рuc. 8. Вектор воды, полученный путем совместного анализа радарного параметра шероховатость и оптического SWB, полученных по снимкам Sentinel-1 за 24 апреля и Landsat-8 за 26 апреля 2017 года для полигона Гастелло, наложенный на маску NDVI

\section{Выводы}

Использование синтезированных данных на точечном уровне позволяет существенно сократить ошибки выделения водной поверхности по радарным или оптическим данным, а на уровне 
объектов совместный анализ позволяет выделить дополнительные классы (затопленная прибрежная растительность, влажные почвы и т.д.).

\section{References}

[1] Arkhipkin O.P., Spivak L.F., Sagatdinova G.N. Development of Flood Space Monitoring in Kazakhstan, Geoscience and Remote Sensing New Achievements. Edited by Pasquale Imperatore \& Daniele Riccio, Vukovar: In Teach, 2010, pp. 419-436. DOI: 10.5772/9113.

[2] Arkhipkin O.P., Sagatdinova G.N. The main results and the directions of development of space monitoring of an emergency in Kazakhstan, Sovremennye Problemy Distantsionnogo Zondirovaniya Zemli iz Kosmosa, 2013, Vol. 10, No. 1, pp. 292-302. (In Russian).

[3] O.P. Arkhipkin, G.N. Sagatdinova. Possibilities of the joint use of optical and radar data in flood space monitoring, The International Archives of the Photogrammetry, Remote Sensing and Spatial Information Sciences, 2018 Vol. 42 (3W4), pp. 67-73. DOI: 10.5194/isprs-archives-XLII-3-W4-67-2018

[4] Arkhipkin O.P., Sagatdinova G.N. Ispol'zovanie polyarimetricheskikh radarnykh dannykh pri kosmicheskom monitoringe pavodkov i navodnenii, Sovremennye Problemy Distantsionnogo Zondirovaniya Zemli iz Kosmosa, 2017, Vol. 14, No. 2, pp. 175-184. DOI: 10.21046/2070-7401-2017-14-2-175-184 (In Russian).

[5] C. Pohl and J. L. van Genderen. Multisensor image fusion in remote sensing: concepts, methods and applications. Int. J. Remote sensing, 1998, Vol. 19, No. 5, pp. 823-854. DOI: 10.1080/014311698215748.

[6] G. Simone, A. Farina, F.C. Morabito, S.B. Serpico, L. Bruzzone. Image fusion techniques for remote sensing applications, Information Fusion, 2002, No. 3, pp. 3-15. DOI: https://doi.org/10.1016/S1566-2535(01)00056-2.

[7] Hassan Ghassemian, A review of remote sensing image fusion methods, Information Fusion, Vol. 32 No. PA, pp.75-89, November 2016. DOI: https://doi.org/10.1016/j.inffus.2016.03.003.

[8] Joshi, N.P., Baumann, M., Ehammer, A., Fensholt, R., Grogan, K., Hostert, P., Jepsen, M.R., Kuemmerle, T., Meyfroidt, P., Mitchard, E.T., Reiche, J., Ryan, C.M., \& Waske, B. A Review of the Application of Optical and Radar Remote Sensing Data Fusion to Land Use Mapping and Monitoring, Remote Sensing, 2016, Vol. 8, p. 70. DOI: https://doi.org/10.3390/rs8010070.

[9] Zakharova L.N., Zakharov A.I., Sorochinskii M.V., Ryabokon' G.P., Leonov V.M. Joint analysis of the data of optical and radar sensors: Potentialities, limitations, and prospects, Radiotekhnika i Elektronika, 2011, Vol. 56, No. 1, pp. 5-19. (In Russian).

[10] V.N. Kopylov, Yu.M. Polishchuk, V.A. Khamedov. Methodological issues for usage space images in rapid assessments impacts of forest fire, Sovremennye Problemy Distantsionnogo Zondirovaniya Zemli iz Kosmosa, 2007, Vol. 4, pp. 155-161. (In Russian).

[11] Chaouch, N., Temimi, M., Hagen, S., Weishampel, J., Medeiros, S., Khanbilvardi, R. A synergetic use of satellite imagery from SAR and optical sensors to improve coastal flood mapping in the Gulf of Mexico, Hydrol. Process. 2012, Vol. 26, pp. 1617-1628. DOI: https://doi.org/10.1002/hyp.8268.

[12] Ejaz Hussain and Jie Shan. Mapping major floods with optical and SAR satellite images, 2010, p. 4, https://engineering.purdue.edu/ jshan/publications/2010/IGARSS_flood_formatted_Hussain_Shan.pdf

[13] Xiaohua Tong, Xin Luo, Shuguang Liu, Huan Xie, Wei Chao, Shuang Liu, Shijie Liu, A.N. Makhinov, A.F. Makhinova, Yuying Jiang. An approach for flood monitoring by the combined use of Landsat 8 optical imagery and COSMO-SkyMed radar imagery, ISPRS Journal of Photogrammetry and Remote Sensing 136, 2018, pp. 144-153. DOI: https://doi.org/10.1016/j.isprsjprs.2017.11.006.

[14] Annarita D’Addabbo, Alberto Refice, Guido Pasquariello, Francesco P. Lovergine, Domenico Capolongo, and Salvatore Manfreda. A Bayesian Network for Flood Detection Combining SAR Imagery and Ancillary Data, IEEE Transactions on Geoscience and Remote Sensing, June 2016, Vol. 54, No. 6, pp. 3612-3625. DOI: 10.1109/TGRS.2016.2520487. 